\title{
Desaster Szenario unter Tage - MED1stMR - neue Ansätze im Training von medizinischen Ersthelfern
}

\author{
Birgit Harthum ${ }^{1}$, Helmut Schrom-Feiertag ${ }^{2}$ und Robert Wenighofer ${ }^{3}$
}

${ }^{1}$ Codoscenter, USECON - The Usability Consultants GmbH, Vienna, Österreich

${ }^{2}$ AIT Austrian Institute of Technology $\mathrm{GmbH}$, Wien, Österreich

${ }^{3}$ Lehrstuhl für Subsurface Engineering, Montanuniversität Leoben, Leoben, Österreich

Eingegangen 16. Oktober 2021; angenommen 5. November 2021; online publiziert 1. Dezember 2021

\begin{abstract}
Zusammenfassung: Medizinische Ersthelfer adäquat und realitätsnahe zu trainieren, ist eine Herausforderung. Realistische Umgebungen, die zu Übungszwecken gesperrt werden können, sind rar gesät und werden oftmals aufgrund von Budget und zeitlichen Einschränkungen selten genutzt. Das Zentrum am Berg (ZaB) der Montanuniversität Leoben, Österreich, hat hierzu bereits 2019 Lösungsansätze entwickelt, die das Training von Blaulichtorganisationen auch in unter Tage Situationen ermöglichen. Die Errichtung von Testzentren wie dem ZaB-Projekt folgt dem Vorschlag der EU-Kommission, die Sicherheit von Straßen- und Eisenbahntunneln zu erhöhen, und ist eine Reaktion auf die mehreren Tausend Tunnelkilometer in der EU, die in Betrieb oder im Bau sind.
\end{abstract}

Mit der Teilnahme des ZaB am Forschungsprojekt MED1stMR wird ein weiterer Schritt in die Verbesserung solcher medizinischen Einsatztrainings unternommen. Nun soll nicht mehr nur im realen Umfeld trainiert werden können, sondern die Erfahrung aus dem Tunnel und seine Besonderheiten für Ersthelfer wandert in die erweiterte virtuelle Umgebung und es soll ein Trainingslösung entstehen, die Virtual Reality (VR) mit haptischen Objekten wie Simulationspuppen und der Messung von Stressdaten der Ersthelfer zu einer Mixed Reality (MR) Lösung vereint, um eine auf künstlicher Intelligenz basierte Szenario Steuerung zu implementieren, um letztlich die Leistung der medizinischen Ersthelfer unter Wahrung ihrer Resilienz zu steigern. MED1stMR Train - [Skills.Resilience.Performance] - Save Lives ist hierzu das Motto des EU geförderten ForschungsProjektes.

\author{
R. Wenighofer ( $\square)$ \\ Lehrstuhl für Subsurface Engineering, \\ Montanuniversität Leoben, \\ Leoben, Österreich \\ Robert.Wenighofer@unileoben.ac.at
}

Schlüsselwörter: Straßentunnel, Eisenbahntunnel, Medizinische Ersthelfer, Einsatzkräfte Training, Virtuelle Welt, Trainingssimulation, Stress, Resilienz, Performance, Wearables, Biosignale, Virtual Reality, Mixed Reality

Disaster Scenario Underground-MED1stMR-New Approaches in Training Medical First Responders

Abstract: Training medical first responders appropriately and realistically is a challenge. Realistic environments that can be closed for training purposes are rare and, due to budget and time constraints, not very often used. The Zentrum am Berg ( $\mathrm{ZaB})$ at the University of Leoben, Austria, developed solutions already in 2019 that enable the training of blue-light organisations in underground situations as well. The establishment of test centres such as the ZaB project follows the EU Commission's proposal to increase the safety of road and railway tunnels and is a response to the several thousand kilometers of tunnels in the EU that are in operation or under construction.

ZaB's participation in the MED1stMR research project is another step towards improving such a medical emergency training. Now it will not only be possible to train in the real environment, but the experience of the tunnel and its specificities for first responders will be transferred to an enhanced virtual environment and a training solution will be created that combines virtual reality (VR) with haptic objects, such as simulation mannequins and the measurement of stress data of first responders, to implement artificial intelligence based scenario control into a mixed reality (MR) solution to ultimately improve the performance of medical first responders while maintaining their resilience. MED1stMR Train-[Skills.Resilience.Performance]-Save Lives is the motto of the EU-funded research project.

Keywords: Road tunnels, Railway tunnels, Medical first responders, First responder training, Virtual world, Training simulation, Stress, Resilience, Performance, Wearables, Biosignals, Virtual reality, Mixed reality 


\section{Einleitung}

Das 3-jährige Projekt (Start Juni 2021) wird im Rahmen des EU Horizon 2020 Programmes mit einer Fördersumme von 7,8 Mio. EUR finanziert. Das Konsortium aus 19 multidisziplinären Partnern aus 9 europäischen Ländern wird vom AIT - Austrian Institute of Technology, Center for Technology Experience angeführt. Partner aus der Wissenschaft und Technologie sowie End Nutzer Partner haben es sich gemeinsam zum Ziel gesetzt, die derzeitigen Trainingsmethoden medizinischer Ersthelfer zu transformieren, um sie besser auf die steigende Anzahl hochkomplexer und unbekannter Krisensituationen vorzubereiten. Abb. 1 gibt einen Überblick über das Projekt.

Es soll eine innovative Mixed-Reality (MR)-Technologie entwickelt werden, die reale medizinische Simulatoren mit virtuellen Umgebungen und intelligenten Szenario Steuerungsoptionen (Smart Scenario Control) kombiniert. Dadurch kann ein bahnbrechendes Training für medizinische Ersthelfer entwickelt werden, welches das Situationsbewusstsein, die Widerstandsfähigkeit und die effektive Leistung während des Einsatzes verbessert. Einer der Projektpartner von MED1stMR ist das Zentrum am Berg (ZaB) der Montanuniversität Leoben, Österreich - eine europaweit einzigartige und unabhängige Forschungseinrichtung rund um den Bau und Betrieb von Untertageanlagen. Durch diese Infrastruktur können im Projekt Trainings zu Forschungszwecken auch real im Tunnelsystem im ZAB stattfinden. Die daraus gewonnenen wissenschaftlichen Ergebnisse werden die Grundlage für das EPME (effective performance in medical emergencies) Modell liefern, das wiederum die Basis für die technologische Lösung bildet (mehr dazu im Abschnitt „Projektvorstellung von MED1stMR“).

\section{Ausgangssituation}

Massenunfälle mit einer großen Zahl von Verletzten nehmen weltweit zu. In solchen Situationen müssen medizinische Ersthelfer Diagnosen stellen und grundlegende lebenserhaltende Maßnahmen ergreifen, um die Opfer zu stabilisieren und am Leben zu erhalten. Bereits diese Situation ist auch für geübte Ersthelfer herausfordernd. Findet der Einsatz in einer besonders ungewohnten Umgebung statt, wie zum Beispiel in einem Tunnel, steigt die
Belastung der Ersthelfer noch mehr und die Performance kann leiden. Tunnels sind Schlüsselelemente der Infrastruktur mit spezifischen Risikoparametern wie der Infrastruktur selbst, den Fahrzeugen und vor allem den Verkehrsteilnehmern. Auf sie sind in Krisenszenarien die Maßnahmen der Ersthelfer gerichtet. Die Vergangenheit um die Jahrtausendwende zeigte die Vulnerabilität von Verkehrsteilnehmern in Unfällen mit Brandentwicklung auf. So verstarben im Jahr 1999 während des Brands im Mont Blanc Tunnel 39 Personen und im Tauerntunnel 12 Personen. Die Unfälle $2001 \mathrm{im}$ Gleinalm Tunnel und dem St. Gotthard Tunnel entsprachen ebenso Krisensituationen in untertägiger Verkehrsinfrastruktur mit ähnlich schrecklichen Auswirkungen. Den Unfällen mit diesen Todeszahlen ist allerdings eine ungleich größere Anzahl verletzter Personen gemeinsam, auf die sich die Ersthelfer konzentrieren müssen [1]. Krisensituationen in Tunnels mit Verletzten gehen jedoch nicht nur auf Brandunfälle zurück. In den Jahren 2006 bis 2012 geschahen in Österreich beispielsweise 768 Unfälle mit Personenschäden mit PKW- und LKW-Beteiligung [2], was die Wichtigkeit des Trainierens kritischer Szenarien durch Ersthelfer in der fremden, die Performance reduzierenden, Tunnelumgebung herausstellt.

Dieser Situation der eingeschränkten Handlungsfähigkeit durch zu hohe Belastung an der Leistungsgrenze kann jedoch entgegengewirkt werden. Entsprechende realitätsnahe Trainings können Ersthelfer auf Situationen und Umgebungen vorbereiten, ihre Resilienz stärken und bessere Lernsituationen schaffen, um die Handlungsfähigkeit der Ersthelfer zu verbessern. Doch Szenarien-basierte realistische Trainings sind oftmals schwierig zu bewerkstelligen. Eine reale Umgebung kann nur selten einfach gesperrt werden. Das ZaB (Abb. 2) am Steirischen Erzberg ist das Tunnelforschungs-, -ausbildungs-, und -trainingszentrum der Montanuniversität Leoben mit jeweils zweiröhrigen Tunnelästen für die Verkehrsträger Straße und Eisenbahn mit jeweils mehreren $100 \mathrm{~m}$ Länge. Es hat hierzu eine reale Trainingsumgebung erstellt, die den exakten Profilen österreichischer Straßen- und Eisenbahntunnels entsprechen. Nicht nur in Bezug auf den Ausbau, sondern auch in Bezug auf die Ausstattung, genutzte Materialien, Lüftung, Beleuchtung etc. sind diese Tunnelsysteme am ZaB real. Einsatzkräfte sind vor allem im ländlichen Raum regelmäßig mit Einsätzen in Tunnels konfrontiert. Daher sind solche Übungen in der Umgebung unter Tage sinnvoll und eine gern gesehene Ergänzung zu normalen Trainings. Real-Trai-
Abb. 1: ÜberblickAusgangssituation und angestrebte Lösung im Projekt

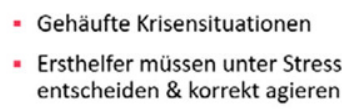

- Gehäufte Krisensituationen

- Ersthelfer müssen unter Stress entscheiden \& korrekt agieren

$$
\begin{aligned}
& \text { - Effektiver Umgang mit Stressoren } \\
& \text { - Realistisches Situationstraining } \\
& \text { - Alternative zu Trainings im realen } \\
& \text { Umfeld finden } \\
& \text { - Resilienz der Ersthelfer }
\end{aligned}
$$
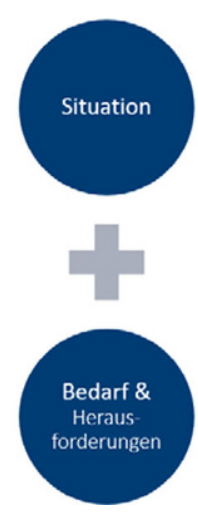

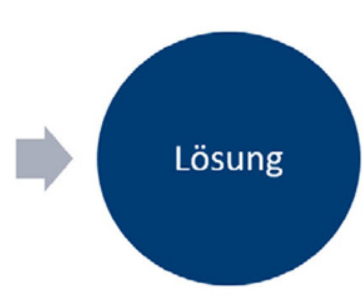

- Mixed Reality Szenario basiertes Trainingssystem - Haptische Simulationspuppen und Sensoren

- Biosignal Messung - smart scenario control

- Steigerung des Selbstvertrauens und der Bewältigungsstrategien von medizin. Ersthelfern 


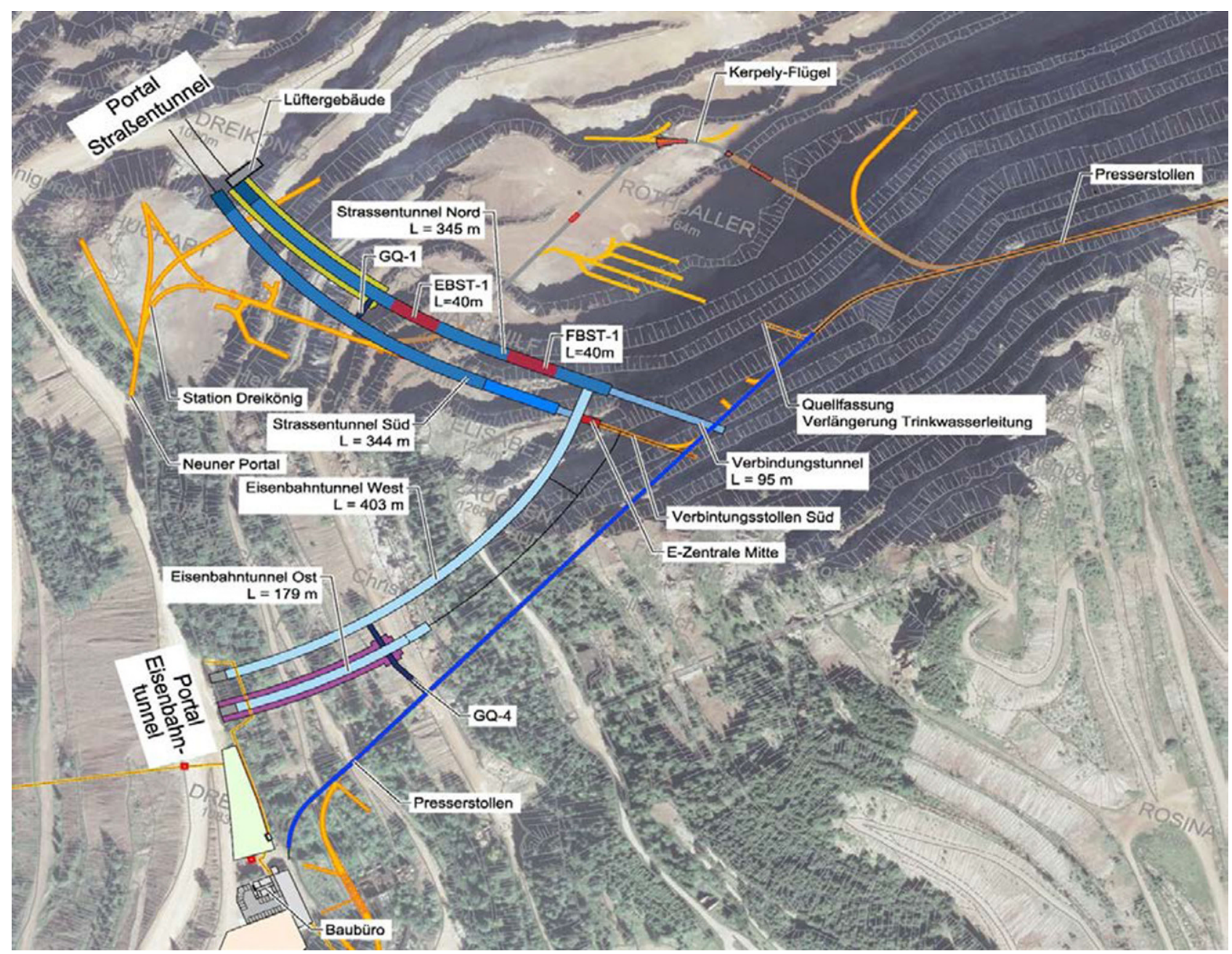

Abb. 2: Tunnelanlage ZaB der Montanuniversität Leoben

ning sind jedoch teuer, und es passiert immer wieder, dass vor allem Anfänger oder in solchen Umgebungen unerfahrene Einsatzkräfte von der echten Erfahrung in einem Tunnel so überfordert sind, dass Trainings abgebrochen werden müssen. Diese zusätzlichen Kosten für einen Neustart eines Trainings sind unvergleichbar hoch. Modernste Trainingsansätze sind es, daher all diese Erfahrung von Einsätzen in eine virtuelle Umgebung zu übertragen und sich dort langsam an die Einsatzerfahrung heranzutasten, bevor in realen Umgebungen trainiert werden kann (Abb. 3).

\section{Lösungsansatz}

Virtual Reality (VR) bringt hier sehr viele Möglichkeiten mit sich und hat gezeigt, dass vor allem bei komplexen Trainingsthemen, die VR das Erfassen und Meistern von neuen Lerninhalten steigert (cf. [3, 4]). Umgebungen (Licht, Höhe, Objekte wie Autos etc.) und Personen (sogenannte Avatare-die animierten Charaktere in einer virtuellen Welt) können jederzeit hinzugefügt und angepasst werden. Zusätzlich können in den Trainingsbedingungen (Unfälle, Lärm, Dunkelheit, verfügbare Ressourcen, etc.) gesteuert werden, um die Trainingseinheiten so realistisch wie möglich zu gestalten. Beim Training medizinischer Fertigkeiten geht es um das Sehen und die Haptik für eine greifbare Interaktion, und wenn eine Simulation nur eines dieser beiden Elemente enthält, bietet sie nur $50 \%$ der Erfahrung. VR wird daher im Projekt MED1stMR zur sogenannten Mixed Reality (MR) erweitert. Denn in der VR ist nahezu alles virtuell - Gegenstände können typischerweise, bis auf ein paar Ausnahmen, nicht haptisch erfasst werden. Bei medizinischen Trainings ist die Haptik jedoch relevant - Personen müssen beatmet, vermessen und analysiert werden - diese Erweiterung der VR Umgebung, um medizinische Simulationspuppen mit realistischer Anatomie erschließen zusätzliche Trainingsmöglichkeiten und bieten eine deutlich höhere Trainingsexperience für den medizinischen Ersthelfer. Zum Beispiel können auslaufende Flüssigkeiten kontrolliert, der Puls direkt an der Simulationspuppe gemessen werden oder eine realistische Beatmung mit medizinischen Utensilien gestartet werden. Die Art der Verletzung wird zusätzlich in der virtuellen Umgebung simuliert und kann jederzeit stärker oder schwächer visualisiert werden. Das bedeutet, die trainierenden Einsatzkräfte sehen eine visualisierte Umgebung und Avatare, fühlen aber die Simu- 


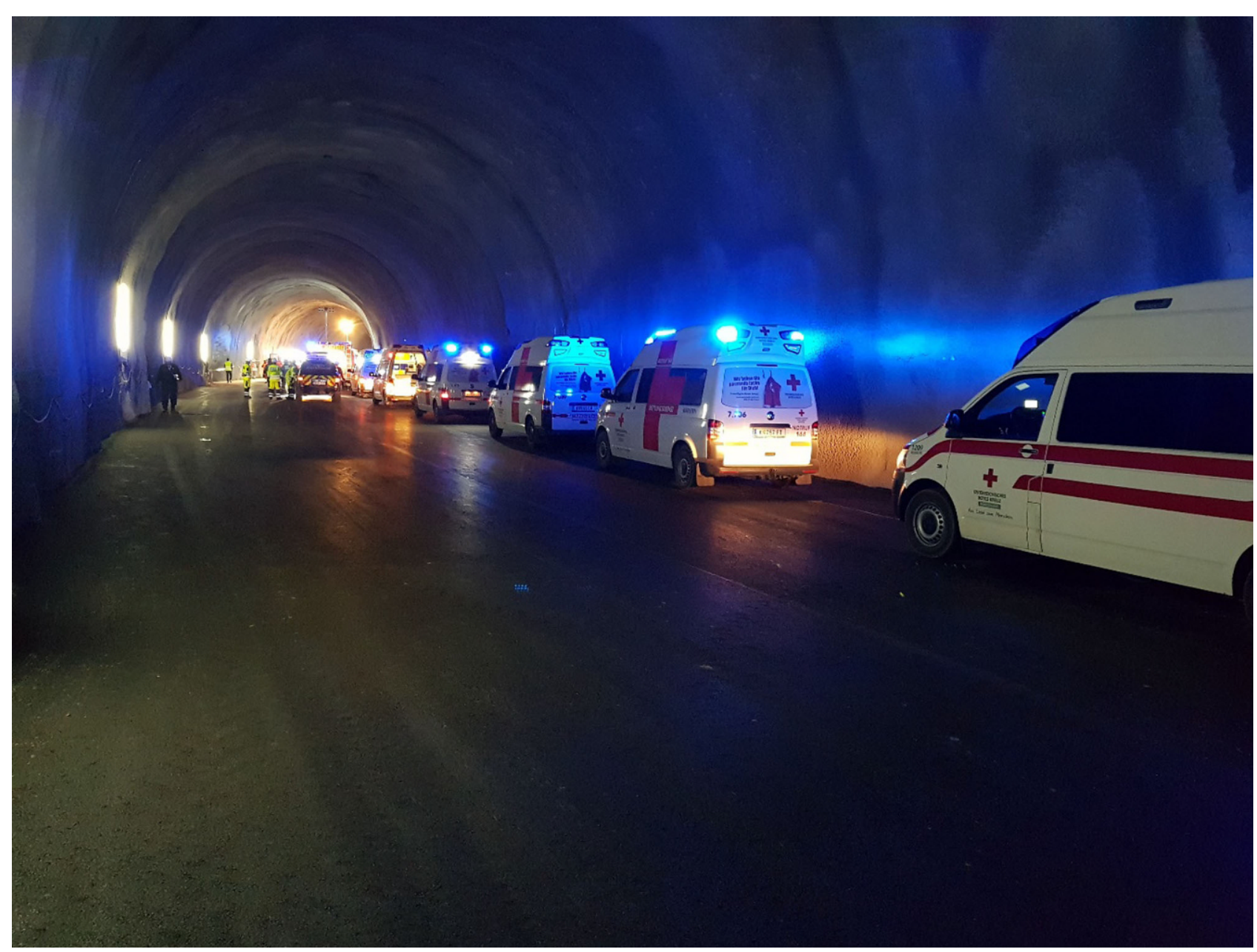

Abb. 3: Untertägige Rettungstraining im Zentrum am Berg (@ Reichhart, 2019-04-14, IRONORE , 2019)

lationspuppe. Dies und die Ergänzung von Objekten, wie z. B. Taschenlampe, Untersuchungsgeräte etc., machen aus einer virtuellen Umgebung eine Mixed Reality (MR). Dies ist aber nur ein Teil der angestrebten Lösung.

Generell hat die bisherige Forschung gezeigt, dass sehr anspruchsvolle und stressige Situationen die Wahrnehmungsfähigkeit, die Entscheidungsfindung und die Handlungsfähigkeit beeinträchtigen. Der stressige Charakter hat einen direkten Einfluss auf die Arbeitsleistung von medizinischen Ersthelfern [5]. Langfristig wirkt sich die mehrfache Exposition gegenüber kritischen Ereignissen, einschließlich physischer und psychischer Stressoren, auch auf die Arbeitszufriedenheit und die psychische Gesundheit von MFR aus [6-8]. Bei der Entwicklung des MR-basierten Trainings wird daher der Fokus auf antizipatorische Affekte, Aufmerksamkeitsprozesse sowie auf Erholungsprozesse und deren Erleichterung bzw. Behinderung der Entscheidungsfindung gerichtet sein. Darüber hinaus soll die Nutzung physiologischer (Biosignale) und verhaltensbezogener Daten (z. B. Bewegungsmuster etc.) zur Steuerung der Szenarien so erfolgen, dass diese Prozesse und Affekte optimal trainiert werden. Im Rahmen des Projekts wird insbesondere der Einfluss dieser kontextuellen und persönlichen Faktoren auf die Wahrnehmung und Bewertung der Situation, die
Entscheidungsfindung und die Leistung der Ersthelfer in hoch komplexen und anspruchsvollen Notfallsituationen untersucht. Das Ergebnis ist ein Modell zur effektiven Leistung in medizinischen Notfällen (EPME-Modell), das im Rahmen des Projekts wissenschaftlich validiert wird. Das EPME-Modell bildet zusammen mit der Stressbewertung die Grundlage für eine KI-basierte intelligente Szenariosteuerung.

Es sollen überdies neuartige tragbare Technologien mit Körpersensoren - smart wearables - entwickelt werden, die nicht nur die physiologischen Stressdaten eines medizinischen Ersthelfers über Biosignale messen, sondern die gewonnenen Daten können auch in das Trainingssystem übertragen und so dem Trainer während des Trainings Auskunft über den Belastungszustand der Trainierenden geben. Durch die Anzeige der Ersthelfer-Belastung mittels errechneter Algorithmen während des Trainings kann der Trainer einerseits manuell das Trainingsszenario an die Bedürfnisse der Ersthelfer anpassen (höhere oder geringere Belastungsfaktoren per Klick auswählen) und dadurch personalisieren. Ein weiterer Nutzen dieser physiologischen Stressmessung stellt andererseits eine daraus resultierende, im Hintergrund automatisiert ablaufende, auf Künstlicher Intelligenz basierte Szenariosteuerung dar. Dieses ad- 
aptive Training wird in der Lage sein, repräsentative Trainingsprogramme und Beurteilungsmöglichkeiten zu schaffen, in denen Kompetenzen in einer integrierten Weise entwickelt werden, d. h. Wahrnehmen, Entscheiden und Handeln treten in gegenseitiger Abhängigkeit auf.

Das EPME Modell kann zusammen mit den Daten über den Zustand und das Verhalten der Trainierenden genutzt werden, um eine Feedback-Schleife für die Personalisierung und Anpassung des Trainings an die Bedürfnisse der Ersthelfer im Training zu bilden, die durch Szenarien auf der Grundlage eines Ansatzes der künstlichen Intelligenz automatisiert wird.

\section{Szenarien}

MED1stMR befasst sich mit aktuellen und zukünftigen Herausforderungen für europäische medizinische Ersthelfer. Für die Effektivität von Ersthelfern wird ein MR Training mit realen Szenarien von großer Bedeutung sein. Um entsprechende Trainingsgerüste zu entwickeln, ist es folglich notwendig, sinnvolle Trainingsszenarien (Abb. 4) zu identifizieren und während der Forschungsarbeit auch im Detail zu analysieren. Tunnel kristallisieren sich aufgrund vieler Faktoren dabei regelmäßig als besonders herausfordernd für Einsatzkräfte heraus. Egal ob Unfälle oder Feuer, im Tunnel herrschen nochmal ganz andere Bedingungen als außerhalb. Die Belastung steigt also allein durch die Umgebung.

Die Tunneltrainingsanlage "Zentrum am Berg“ des MEd1stMR Partners Montanuniversität Leoben bietet die idealen Voraussetzungen, eine Tunnel-Notfallübung durchführen, die Erkenntnisse in das MED1stMR System zu überführen und entsprechende Übungen in der MR Lösung zu erstellen. Letztlich sollen auch Vergleichsstudien dazu gemacht werden. Während der gesamten Vergleichsübung wird die EPME-Leistung der Teilnehmer, der Testgruppe und der Kontrollgruppe aufgezeichnet, um das EPME-Verhalten der medizinischen Ersthelfer und den vermuteten Einfluss einer optimalen Vorbereitung und des Trainings im Vorfeld zu analysieren.

\section{Projektmethode}

Hervorzuheben ist bei MED1stMR jedoch auch die genutzte Projektmethode, für die sich das Konsortium bereits bei der Einreichung des Projektes entschieden hat. Umfangreiche Erfahrungen im Projektgeschäft von Projektleiter AIT und beteiligtem Projektpartner USECON haben gezeigt, dass die Einbindung von Endnutzern bei der Entwicklung eines Produktes oder Services den Erfolg bei der Einführung enorm steigert. Diesen Grundsatz der User Experience aus der Human Computer Interaction Forschung haben sich die Projektkoordinatoren zum Ziel genommen und die

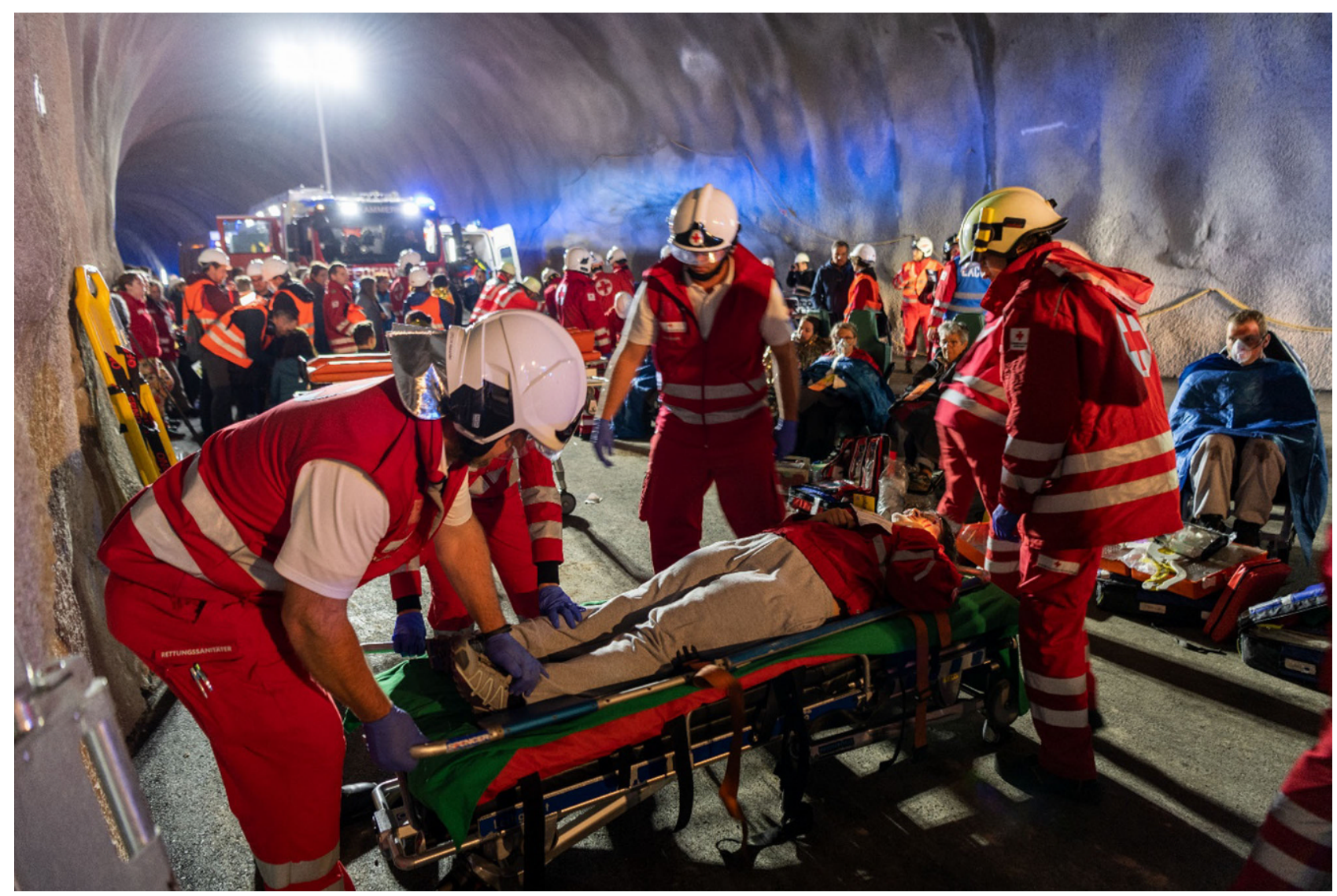

Abb. 4: Bergung verletzter Personen bei Rettungstraining im Zentrum am Berg 
sogenannte AEUCR (Agile End User Centred Research) Methode entwickelt. Hierbei geht es darum, den Endnutzer (also Trainer, Einsatzkräfte und aber auch Entscheider bei der Einführung neuer Trainingssysteme) agil, iterativ und durchgehend in die Projektimplementierung einzubinden. Das bedeutet, dass Endnutzer nicht zu Beginn ihre Bedürfnisse formulieren und am Ende des Projektes eine finale Lösung präsentiert bekommen, sondern durchgehend per definiertem Prozess eingebunden sind. Endnutzer in MED1stMR erarbeiten gemeinsam mit den Forschungsund Entwicklungspartnern realistische Trainingsszenarien, sind Teilnehmer bei wissenschaftlichen Studien und liefern dadurch wertvollen Input und sind Teil regelmäßiger Feedback Loops mit inkrementellen Entwicklungsschritten der technologischen Lösungen.

Das relevante ist dabei natürlich die Erfahrung der Endnutzer und der Trainingsorganisationen. Die Erfahrung des ZaB bei der Erstellung realitätsnaher Tunnelszenarien ist dabei unerlässlich. Die Bedingungen unter Tage sind für ungeübte Ersthelfer eine große Herausforderung und sollten im Trainingsprogramm verankert sein.

\section{Ziele und Nutzen}

Das Hauptziel von MED1stMR besteht darin, medizinische Ersthelfer besser auf stressige und hochkomplexe Katastrophensituationen vorzubereiten, indem eine neue Generation von MR-Training mit haptischem Feedback für mehr Realismus entwickelt wird. MED1stMR hat sich in dem Zusammenhang vier Ziele (Abb. 5) gesetzt. Einerseits die beiden technologischen Ziele einer MR Lösung sowie dem Smart Scenario Control zur KI-basierten automatisierten Steuerung von Szenarien aufgrund der Biosignale der Trainierenden, aber auch das Ziel, ein Trainingsgerüst für medizinische Ersthelfer zu erstellen, dass aufgrund der Analyse bekannter Trainingsmethoden und der Ergebnisse aus dem Projekt die Eingliederung in bestehende Trainings ermöglicht. Das Thema medizinischer Ersthelfertrainings und aller damit im Zusammenhang stehenden Ergebnisse sollen auBerdem europaweit kommuniziert werden und Nutzen für Netzwerke und moderne Ansätze in der Aus- und Fortbildung der medizinischen Ersthelfer stiften.

Im Zuge der Szenarien Erstellung komplexer Umgebungen in der MR Lösung ist es demnach auch Ziel den Tunnel ein stückweit in die virtuelle Umgebung zu bringen, um solch ungewohnte und belastende Umgebungen einer breiteren Masse an Einsatzkräften zugänglich zu machen. Es macht auch aus ökonomischer Sicht Sinn, Ersthelfer nicht sofort mit Übungen im echten Tunnel und dadurch eventu- ell bedrohlichen Übungen zu überfordern. Im MED1stMR System kann das gemeinsam mit den Partnern erstellte Szenario schrittweise mit Stressfaktoren erweitert werden (z. B. mehr oder weniger Licht im Tunnel, höhere oder geringere Sichtweite durch virtuellen Rauch oder mehr oder weniger verletzte Personen) und dadurch eine hochflexible Trainingsumgebung darstellen.

\section{Zusammenfassung}

Das EU Horizon 2020-Forschungsprojekt MED1stMR hat zum Ziel, derzeitige Trainingsmethoden von medizinischen Ersthelfern zu transformieren und sie dadurch besser auf stressige und hochkomplexe Katastrophensituationen vorzubereiten. Es wird eine innovative Mixed-RealityTechnologie (MR) entwickelt, um reale medizinische Simulatoren mit virtuellen Umgebungen zu kombinieren. Im Rahmen des Projekts werden Trainingsmethoden für medizinische Ersthelfer in der MR-Umgebung entwickelt, um ihr Situationsbewusstsein, ihre Widerstandsfähigkeit und ihr effektives Verhalten bei medizinischen Notfällen in hochkomplexen und unvorhersehbaren Situationen zu verbessern. Szenarien in Tunnels helfen hierbei, spezielle Situationen bereits vorab erfahren zu können. Die Sperrung eines Tunnels über lange Zeit verursacht enorme Kosten. Diese durch effizientere Einsätze mindern zu können, ist ein relevanter Faktor bei der Erstellung von neuen Trainingsmethoden.

Das Verhalten, die Interaktion und die Bedingungen unter Tage auf diese Weise auch größeren und weiter entfernten Einsatzkräften zugänglich zu machen, ist ein Aspekt dieses Forschungsprojektes. Einen Überblick über die anderen Aspekte zum Projekt gibt es unter www.med1stmr. eu.

Übersicht der Projektpartner:

- AIT - Austrian Institute of Technology GmbH - Center for Technology Experience

- Ruprechts-Karls-Universität Heidelberg

- Umeå University

- Universität Bern

- Montanuniversität Leoben - Department Zentrum am Berg

- Refense AG

- Plux - Wireless Biosignals S.A.

- D2D Holding BV

- IDENER SCIENTIFIC COMPUTING

- Usecon - The Usability Consultants GmbH

- Mindconsole Gmbh

Abb. 5: Projektziele MED1stMR

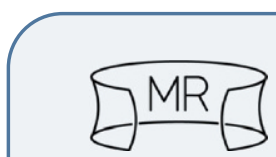

Mixed Reality Lösung

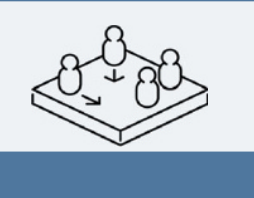

Trainings Gerüst

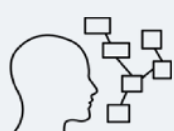

Smart Scenario

Control

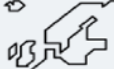
(1)

Europaweite

Positionierung 
- Simcampus Zentrum GmbH

- Hellenic Rescue Team

- Johanniter Österreich

- Servicio Madrileno De Salud

- Universitatsklinikum Heidelberg

- Region Jämtland Härjedalen

- Johanniter International

- Campus Vesta

Förderung. Dieses Projekt wird im Rahmen des Horizon 2020 Forschungsund Innovationsprogramms der Europäischen Union gefördert (Fördervertrags Nr. 101021775). Der Inhalt gibt ausschließlich die Meinung des MED1stMRsKonsortiums wieder. Die Europäische Exekutivagentur für die Forschung und die Europäische Kommission haften nicht für die Richtigkeit der hierin enthaltenen Informationen.
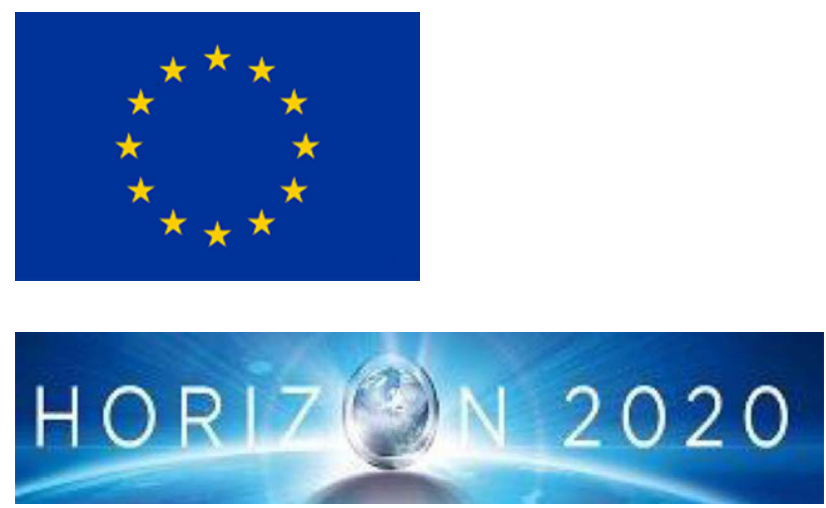

Funding. Open access funding provided by Montanuniversität Leoben.

Open Access Dieser Artikel wird unter der Creative Commons Namensnennung 4.0 International Lizenz veröffentlicht, welche die Nutzung, Vervielfältigung, Bearbeitung, Verbreitung und Wiedergabe in jeglichem Medium und Format erlaubt, sofern Sie den/die ursprünglichen Autor(en) und die Quelle ordnungsgemäß nennen, einen Link zur Creative Commons Lizenz beifügen und angeben, ob Änderungen vorgenommen wurden.
Die in diesem Artikel enthaltenen Bilder und sonstiges Drittmaterial unterliegen ebenfalls der genannten Creative Commons Lizenz, sofern sich aus der Abbildungslegende nichts anderes ergibt. Sofern das betreffende Material nicht unter der genannten Creative Commons Lizenz steht und die betreffende Handlung nicht nach gesetzlichen Vorschriften erlaubt ist, ist für die oben aufgeführten Weiterverwendungen des Materials die Einwilligung des jeweiligen Rechteinhabers einzuholen.

Weitere Details zur Lizenz entnehmen Sie bitte der Lizenzinformation auf http://creativecommons.org/licenses/by/4.0/deed.de.

\section{Literatur}

1. Haack, A.: Current safety issues in traffic tunnels, Tunnelling and Underground Space Technology, 17 (2002), pp 117-127

2. ILF Consulting Engineers: Auswertung der ASFINAG-Tunnelbrandstatistik 2006-2012, RVS-Arbeitsausschuss 09.03.11 TuRisMo, (2013), pp 1-38

3. Huang, H. M.; Rauch, U.; Liaw, S. S.: Investigating learners' attitudes toward virtual reality learning environments: Based on a constructivist approach, Computers \& Education, 55 (2010), pp 1171-1182

4. Bossard, C.; Kermarrec, G.; Buche, C.; Tisseau, J.: Transfer of learning in virtual environments: A new challenge?, Virtual Reality, 12 (2008), pp 151-161

5. Regehr, C.; Leblanc, V. R.: PTSD, acute stress, performance and decision-making in emergency service workers, Journal of the American Academy of Psychiatry and the Law, 45 (2017), pp 184-192

6. Arble, E.; Arnetz, B. B.: A Model of First-responder Coping: An Approach/Avoidance Bifurcation, Stress and health: journal of the International Society for the Investigation of Stress, 33 (2017), pp 223-232

7. Frenkel, M. O.; Giessing, L.; Egger-Lampl, S.; Hutter, V.; Oudejans, R. R. D.; Kleygrewe, L.; Jaspaert, E.; Plessner, H.: The impact of the COVID-19 pandemic on European police officers: Stress, demands, and coping resources, Journal of Criminal Justice, 72 (2021), p 101756

8. Olatunji, S.: Understanding the Perceptions and Decision-Making Behaviors of First Responders in the Context of Traumatic Events and PTSD, Walden Dissertations and Doctoral Studies, 2018

Hinweis des Verlags. Der Verlag bleibt in Hinblick auf geografische Zuordnungen und Gebietsbezeichnungen in veröffentlichten Karten und Institutsadressen neutral. 